\title{
General overview on sleep medication
}

\author{
Floriana Boghez ${ }^{1}$, loana Mindruta ${ }^{2}$ \\ ${ }^{1}$ Academica Clinic, Bucharest, Romania \\ Emergency University Hospital of Bucharest, Romania
}

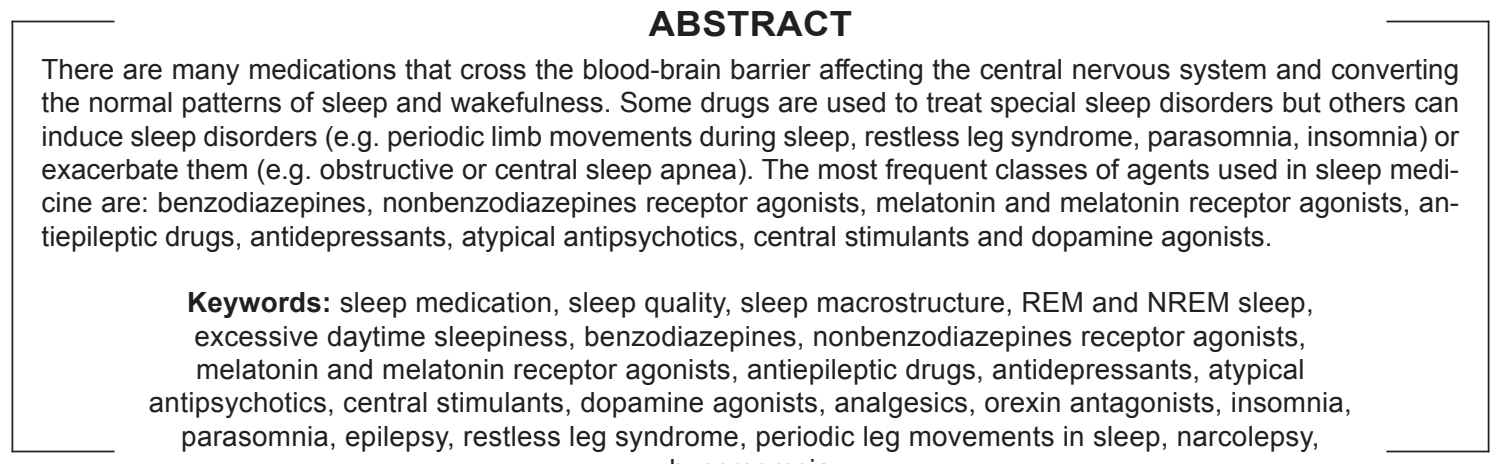
hypersomnia

The prevalence of sleep disorders (more than 80 different disorders) is augmenting in our societies where constant exposure to artificial light (television, computers, mobile-phones), interactive activities, socioeconomic pressure and needs drive us into a 24-hour society ("a world that never sleeps"), where the link between work and social time is broken and the sleep health can be compromised. Insomnia and excessive daytime sleepiness are the most common symptoms in sleep medicine practice and sometimes we need medication to improve at least the quality of life of these patients, if we cannot remove the cause of them.

Any medication that passes through the bloodbrain barrier has the potential to alter the quality and/or architecture of sleep. Sleep quality is the degree to which restful and restorative sleep is maintained during the night and a subjective assessment about the way the individual feels refreshed on waking and on functioning throughout the day. We objectively appreciate the sleep quality with: latency until sleep onset (SO), wakefulness after sleep onset (WASO) and the duration of sleep (usually on polysomnographic recordings). Sleep architecture represents the macrostructure of sleep. Sleep is cyclical, with each cycle composed several non-REM sleep stages (N1, N2 and N3) and rapid eye movement (REM), assessed primarily by electroencephalography (EEG) patterns during polysomnography. Each sleep drug can modify the sleep quality and the sleep macrostructure/microstructure and the ideal sleep pill should increase the sleep quality and maintain the physiologic sleep cycles. In this article, we are going to review the most frequent drugs used in sleep disorders.

\section{Benzodiazepines}

Benzodiazepines (Diazepam, Lorazepam, Midazolam, Alprazolam, Clonazepam) and nonbenzodiazepine receptor agonists (Zolpidem, Zaleplon, Eszopiclone) are commonly prescribed sedative-hypnotic medications used to treat insomnia.

Gamma-aminobutyric acid (GABA) is the major inhibitory neurotransmitter in the brain and ex- 
erts its effects via ionotropic and metabotropic receptors. $\mathrm{GABA}_{\mathrm{A}}$ and $\mathrm{GABA}_{\mathrm{C}}$ receptors are linked directly to chloride ion channels, whereas $\mathrm{GABA}_{\mathrm{B}}$ receptors are $G$ protein coupled. Many drugs that cause sleep or loss of consciousness enhance transmission at the $\mathrm{GABA}_{\mathrm{A}}$ receptor. Benzodiazepines facilitate GABA-mediated inhibition of cell firing by binding to a subunit of the $\mathrm{GABA}_{\mathrm{A}}$ receptor complex called the benzodiazepine receptor $(1,2,3)$.

They improve sleep quality by decreasing the sleep latency (SL), increasing the total sleep time (TST) and decreasing WASO (2). The sleep architecture does not always remain the same: for example, the benzodiazepine can be a suppressor of $\mathrm{N} 3$ sleep and at high doses of REM sleep (1). They reduce N1 (which is a positive effect) but increase N2 and the spindle activity during this sleep stage (the frequency and amplitude of sleep spindles) $(6,7)$. Benzodiazepines can depress respiration and should be used cautiously in patients with sleep apnea or hypoventilation $(1,2)$. They reduce the severity of symptoms in patients with restless legs syndrome (3).

The action duration of benzodiazepine is important in deciding when to use them: short-acting agents (Alprazolam, Midazolam, Triazolam, Oxazepam), intermediate-acting agents (Clonazepam, Lorazepam) and long-acting agents (half-life values usually exceed 24 hours: Diazepam, Clobazam, Flurazepam) $(1,2)$.

Their use comes with several side effects: daytime sleepiness, poor motor coordination, increase in falls, cognitive impairment, confusion and risk of dependency (4). Patients typically develop tolerance to these agents and with chronic use, higher dosages may be required. Discontinuation of the treatment can induce a rebound insomnia and a rebound of REM sleep (3).

Benzodiazepines can be used as antiepileptic drugs, especially in patients with frequent nocturnal seizures (Clonazepam in childhood epilepsies or pharmaco-resistent epilepsies, Clobazam in Lennox-Gastaut syndrome and Landau-Kleffner syndrome, Lorazepam or Diazepam iv. in status epilepticus) (5). They are not usually the first choice for seizure prevention and they tend to be additional anticonvulsive treatment. On the other hand, they tend to increase the percent of N2 which is the main stage of sleep when seizures appear (5).
Clonazepam is the first line treatment in patients with REM sleep behavior disorder (RBD) but should be used with caution in patients with dementia, gait problems or concomitant sleep apnea $(8,10)$. It is the second line treatment in refractory restless leg syndrome (RLS) (9). It can be also used for sleep starts, hypnagogic foot tremor, propriospinal myoclonus at sleep onset and excessive fragmentary myoclonus (when it is the sole abnormality in patients with EDS) $(1,3)$.

\section{Nonbenzodiazepine receptor agonists}

Apparently, the nonbenzodiazepine receptor agonists (Zolpidem, Zaleplon, Eszopiclone), acting via the $\mathrm{GABA}_{\mathrm{A}}$ receptor complex (at a different site than the benzodiazepines) do not affect the amount of N3 and do not affect breathing during sleep $(1,11)$. They decrease sleep latency and WASO and they might have minimal effect on REM sleep (12). Tolerance and withdrawal effects are not so frequent as for the benzodiazepines (13).

They are indicated for short-term management of insomnia and small doses are recommended for older patients. Adverse effects may include impaired alertness and motor coordination. Compared to conventional benzodiazepines, they are not muscle relaxants, anticonvulsants or anxiolytics. The usual dose of Zolpidem (the most used drug of this category) is $5-10 \mathrm{mg} /$ day and women are seemingly slower metabolizers of Zolpidem than men (13).

The American Geriatrics Society (AGS) Beers Criteria identifies Zolpidem as a potentially inappropriate medication for the elderly due to concerns for the adverse effects mentioned and the risk for delirium, falls and fractures (14).

There is conflicting evidence as to whether Zolpidem is associated with rebound insomnia $(1,3)$. Another concern to clinicians is the occurrence of complex sleep-related behaviors (CSBs) (15). The use of Zolpidem, Zopiclone and Zaleplon has been associated with certain CSBs, including sleepwalking with object manipulation (e.g., cooking, cleaning), sleep conversations (on the telephone or in person), sleep eating, sleep driving, sleep shopping, and sleep sex $(15,16)$.

\section{Melatonin and melatonin receptor agonists}

Considering the function of suprachiasmatic nucleus in circadian rhythm regulation, melatonin and 
melatonin agonists can be used as a treatment for circadian rhythm sleep disorders and insomnia. Melatonin (a pineal gland hormone, released mostly during darkness) binds to all three types of melatonin receptors and as a drug, it is a good chronobiotic agent used for phase shifting circadian rhythm (a first-line treatment for this sleep problems) (1-3). Melatonin increases sleep propensity when taken in the evening and can produce a phase delay when administered in the morning (17).

Ramelteon (a melatonin receptor agonists) binds to melatonin receptors type 1 and 2 (MT1 and MT2), inhibiting the activity of suprachiasmatic nucleus in promoting wake. It has no direct sedative effects. In contrast to Melatonin, it has lower sensitivity for MT3. It is used for the treatment of sleep onset insomnia $(18,19)$.

\section{Antiepileptic agents}

By their many mechanisms of action (blocking the voltage-dependent sodium channels, blocking the glutamate receptors, stimulating GABA-ergic effects, inhibiting GABA degradation, modulating calcium channels, decreasing potassium efflux, inhibiting the glutamate release etc.), antiepileptic drugs (AED) can modify sleep, with beneficial or detrimental effects (5).

Carbamazepine may increase slow wave sleep and but decreases REM sleep and there are studies which demonstrated the initial administration of Carbamazepine leading to an increased number of sleep stages shifts and increasing fragmentation of REM sleep $(20,21)$. In the Gigli's study (initiation of CBZ treatment in seven patients with temporal lobe epilepsy), it seems that disruption of REM sleep was present only in the acute phase of drug administration. Looking for REM sleep disorders, in our own practice, we met a patient with severe nightmares after the initiation of Carbamazepine (monotherapy for seizures).

Valproate's effects are variable, with some patients showing sleep stabilization and others showing increased arousals $(20,22)$.

Gabapentin increases REM sleep and slow wave sleep duration and decreases the awakenings and the N1 stage $(20,22)$. It is the only AED shown to be effective for periodic limb movements in sleep $(1,3)$.

Somnolence was reported in $30 \%$ of patients taking Pregabalin and this adverse effect seems to be mild or moderate in intensity, occurring within the first two weeks of therapy initiation (24).

Mildly detrimental effects on sleep are possible for Ethosuximide because it increases $\mathrm{N} 1$ and decreases N3 $(20,22)$.

Lamotrigine has been reported to cause insomnia and the insomnia appeared to be dose-related (23). On the other hand, drowsiness is one of the most frequent side-effects and is dose-dependent too, probably because it increases REM sleep and decreases the amount of slow-wave sleep (15).

Epileptic patients taking Levetiracetam proved to develop a sleep consolidation (increase in total sleep time spent in NREM stages) while volunteers were less alert and sleepier on waking (26).

Add-on trials reported a higher frequency of somnolence in patients receiving Topiramat (29) while other studies showed no daytime sleepiness on starting doses of $200 \mathrm{mg} / \mathrm{d}$ (28). This drug can be a second-line treatment for sleep-related eating disorder (27) (SSRIs are the first line).

Subjective somnolence was reported in patients taking Vigabatrin in the first 4-6 weeks after drug initiation (30). The Vigabatrin-administered kindled rats showed an increase in total sleep time due to an increase in total N1 sleep stage (31).

Lacosamide $300 \mathrm{mg} /$ day had no effect on objective or subjective sleep parameters in healthy subjects and was generally well tolerated $(32,33)$.

For patients with frequent nocturnal seizures, AEDs can also stabilize sleep by decreasing seizures and interictal epileptiform discharges. Newer generation of AEDs generally tend to cause less disturbance of the sleep cycle and greater stabilization of sleep architecture.

\section{Antidepressants}

Antidepressant agents may influence sleep: some are sedating and some are stimulating.

The tricyclic agents (TCA) act by blocking the serotonin and norepinephrine transporters. Tertiary amines (Amitriptyline, Clomipramine, Doxepin, Imipramine) induce excessive daytime somnolence (EDS) and impairment of cognitive and psychomotor performances. They can be use in insomniac patients (34). Secondary amines (Desipramine, Protriptyline) are activating antidepressants by increasing the sleep latency and WASO with a lower sleep efficiency (35). TCAs can be used to treat re- 
sistant insomnia but they can cause abnormal dreams. Sudden drug withdrawal can produce REM sleep rebound and insomnia.

Selective serotonin reuptake inhibitors (SSRIs) are the most commonly prescribed antidepressants. SSRIs (Paroxetine, Fluoxetine, Sertraline, Escitalopram) block the reabsorption (reuptake) of the serotonin in the brain. They can cause EDS (Paroxetine, Fluvoxamine) or insomnia (Citalopram, Fluoxetine) (1,2,3). SSRIs can precipitate or worsen periodic limb movements of sleep and restless legs syndromes and the effect might be dose-dependent (36,37). They can also increase other movement disorders during sleep and REM sleep behavior disorder (RBD) and can amplify the electromyographic activity during REM sleep (38). Abnormal slow eye movements during sleep has been observed after ingestion of Fluoxetine ("Prozac eyes") (39).

Venlafaxine is a serotonin and norepinephrine reuptake inhibitor (SNRI) used as antidepressant and anxiolytic, but also for the treatment of episodes of cataplexy. It can cause insomnia or EDS and may increase the periodic leg movements of sleep (37). Status cataplecticus (a rare manifestation of narcolepsy with cataplexy episodes recurring for hours or days, without a refractory period, in the absence of emotional triggers) can be developed after abrupt onset of Venlafaxine $(40,41)$. Orexins excite neurons of the locus coeruleus and dorsal raphe nucleus; drugs that increase noradrenergic and serotoninergic transmitters suppress cataplexy $(66,67)$. But then, treatment of cataplexy with antidepressants may trigger or exacerbate RBD symptoms.

Serotonin antagonist and reuptake inhibitors (SARI) block the serotonin receptors and inhibit the reuptake of serotonin, norepinephrine and dopamine. Trazodone is a sedating antidepressant which increases the N3 stage of sleep and decreases the REM stage $(42,43)$. Instead, Nefazodone decreases N3 and increases N2 (44).

Monoamine oxidase inhibitors (MAOI), the first developed type of antidepressants, which inhibit the enzymes involved in the metabolism of norepinephrine, dopamine and serotonin, usually produce insomnia but may also induce EDS. They decrease the total sleep time, increase WASO and decrease REM sleep (45). MAOI's are the most potent REM inhibitors and REM sleep rebound can occur frequently after drug withdrawal. MAOI (Phenelzine, Selegiline, Tranylcypromine, Rasagiline) can cause abnormal dreams and nightmares.

In conclusion, almost all antidepressant agents suppress REM sleep (increase the REM latency and decrease the amount of REM sleep) but their effect on sleep latency and sleep quality is different and some studies are contradictory.

\section{Atypical antipsychotics}

Atypical antipsychotic medications are often used as hypnotics. Whereas the typical antipsychotic medications have only dopaminergic antagonism, the atypical antipsychotic medications have both serotonergic and dopaminergic antagonism. Quetiapine, Olanzapine, Clozapine, Risperidone reduce the sleep latency and WASO and increase the total sleep time $(46,47,48)$. In general, they suppress REM sleep and increase N3. Because of their long half-time, diurnal sedation is frequent. Quetiapine and Risperidone might cause or increase periodic leg movements of sleep and restless leg syndrome (49).

\section{Central nervous system stimulants}

Medication that stimulates the central nervous system (CNS) has important effects on sleep quality and quantity. Modafinil, Methylphenidate and Dextroamphetamine may be used in sleep disorders with somnolence (narcolepsy, hypersomnia etc.) $(3,4)$. They increase sleep latency and wakefulness during sleep period, increase N1 and decrease N3 and REM. Usually patients develop tolerance to these side effects on sleep. Stopping the medication can induce REM rebound and EDS. The amphetamines (Methylphenidate, Dextroamphetamine, Methamphetamine) act by increasing the synaptic availability of norepinephrine and dopamine $(1,4)$. But there are several problems with their use. First, tolerance may develop requiring escalating doses and sometimes "a drug holiday" (several days without medication) may be useful. Second, they increase blood pressure. Third, they induce other side effects: nervousness, irritability, headache, decreased appetite, insomnia, paranoia or hallucinations.

The mechanism of Modafinil remains elusive but it seems to have monoaminergic effects stimulating the histamine, norepinephrine, dopamine, 
serotonin and orexin systems $(50,51,52)$. Modafinil may reduce the effectiveness of birth control medications (53). It can be use in somnolence in relation to narcolepsy, idiopathic hypersomnia, periodic limb movement disorder, Parkinson disease, obstructive sleep apnea, multiple sclerosis or myotonic dystrophy $(1,4)$.

\section{Other medications used in narcolepsy and hypersomnia}

Sodium oxybate (Gamma-hydroxybutyrate), a GABA precursor, increases sleep continuity and decreases the frequency of cataplexy in persons with narcolepsy $(54,55)$. Gradual and mild improvement in EDS has also been reported with chronic use. Adverse effects include enuresis, parasomnia, headaches, dizziness, vertigo and nausea. Over dosage can result in respiratory depression, confusion, seizures, coma or death. It is usually taken twice each night because it has a very short half-time (using an alarm clock for the second dose is an important inconvenient of this drug). The precise mechanism by which sodium oxybate reduces cataplexy is not known yet. It is contraindicated in patients treated with sedative hypnotics (4).

Pitolisant is a histamine H3 antagonist, a new wake-enhancing drug that increases the histamine release in the brain by blocking presynaptic $\mathrm{H} 3$ histamine reuptake, a drug that can improve the EDS in hypersomnia (idiopathic and symptomatic) and narcolepsy (56).

\section{Dopamine agonists}

Dopaminergic agonists (Pramipexol - D2 and D3 receptor agonist, Ropinirole - with high specificity for D3 receptors) represents the first line of sleep treatment in periodic limb movements of sleep (PLMS) and restless leg syndrome (RLS) $(57,58,59)$. The most frequent side effects are nausea and somnolence, which typically decrease over time. Rotigotine can be used as a transdermal patch. PLMS should be treated when it is associated with RLS, insomnia or EDS. An unusual but interesting adverse effect of dopamine agonist is the pathologic gambling (60).

There are some other drug categories that can be efficient in PLMS: AEDs (Gabapentin, Pregabaline, Valproate), opioids, iron supplementation and benzodiazepines (Clonazepam) $(1,4)$.

\section{Analgesics}

The most used analgesics are the nonsteroidal anti-inflammatory drugs (NSAIDs), the antipyretics (Acetaminophen) and the opioids (Codeine, Morphine).

Acetaminophen doesn't change sleep quality and architecture at therapeutic doses (61).

There are some studies who say that NSAIDs can modify sleep by inhibiting prostaglandin synthesis which is believed to promote sleep (61).

Opioids decrease N3 and REM at high dosages and among addicted persons increase WASO and decrease total sleep time $(62,63)$. Central sleep apnea can arise from chronic use of long-acting opioids (Methadone, Hydrocodone, Morphine). They may also produce ataxic breathing during sleep (Biot's breathing). On the other hand, they may improve sleep significantly in patients with chronic severe pain (64).

\section{Orexin antagonists}

A new class of hypnotics is in phase of development. Orexin receptors are distributed widely in the brain and their activation promotes wakefulness. Two neuropeptide orexins have been identified, $\mathrm{OX} 1$, mainly present in locus coeruleus, and OX2, expressed mainly in hypothalamic histaminic areas (3). Insertion of an antagonist (Suvorexant) suppresses the arousal and these drugs are now used in trails with patients with primary insomnia (25).

\section{Prazosin}

It is a selective alpha-1-adrenergic antagonist and it is now considered a first-line drug for the treatment of nightmares of posttraumatic stress disorder (68).

\section{Menopausal hormone replacement therapy}

Sleep problems are frequent in menopausal and perimenopausal women. Aging, weight gain, depression, vasomotor instability, sleep apnea, fibromyalgia can represent one or multiple causes of poor sleep at these patients. Menopausal hormone replacement therapy (HRT) may improve sleep structure and quality (1-3).

Progesterone is a GABA agonist with anxiolytic and sedative effects. It has a stimulating effect on breathing with some efficiency in mild obstructive sleep apnea in menopausal women $(69,71,72)$. 
Estrogen promotes sleep by decreasing sleep latency, reducing the number of arousals and awakenings and increasing the amount of slow-wave sleep and REM. It is a serotonin and GABA agonist $(70,72)$.

\section{CONCLUSION}

From sleep medicine point of view, when we manage patients with neurological diseases and sleep problems, we bear in mind not only inducing

\section{REFERENCES}

1. Kryger M.H., Roth T., Dement W.C. Principles and Practices of Sleep Medicine, 5th ed - Elsevier Saunders, St. Louis, MO 2011

2. Teofilo Lee-Chiong Jr., Sleep Medicine: essentials and reviews - Oxford University Press, 2008

3. Claudio Bassetti, Zoran Dogas, Philippe Peigneux, Sleep Medicine Textbook - 2014, European Sleep Research Society

4. Christian Guilleminault, Excessive Sleepiness - Sleep Medicine Clinics, 2006

5. C.W. Bazil, B.A. Malow, M.R. Sammaritano, Sleep and Epilepsy: the clinical spectrum - Elsevier, 2002

6. Bastien C.H., LeBlanc M., Carrier J., Morin C.M. Sleep EEG power spectra, insomnia, and chronic use of benzodiazepines - Sleep 2003 May 1;26(3):313-7

7. Suetsugi M., Mizuki Y., Ushijima I., Kobayashi T., Watanabe Y. The effects of diazepam on sleep spindles: a qualitative and quantitative analysis - Neuropsychobiology. 2001 Jan;43(1):49-53

8. Ferri R., Marelli S., Ferini-Strambi L., Oldani A., Colli F., Schenck C.H., Zucconi M. An observational clinical and video-polysomnographic study of the effects of clonazepam in REM sleep behavior disorder - Sleep Med, 2013 Jan 14(1):24-9

9. Rinaldi F., Galbiati A., Marelli S., Ferini Strambi L., Zucconi M. Treatment Options in Intractable Restless Legs Syndrome/ Willis-Ekbom Disease - Curr Treat Options Neurol, 2016 Feb 18(2):7

10. R. Nisha Aurora, Rochelle S. Zak, Rama K. Maganti, Sanford H. Auerbach, Kenneth R. Casey, Susmita Chowdhur, Anoop Karippot, Kannan Ramar, David A. Kristo, Timothy I. Best Practice Guide for the Treatment of REM Sleep Behavior Disorder Morgenthaler, Journal of Clinical Sleep Medicine, Vol.6, No. 1, 2010

11. McCann C.C., Quera-Salva M.A., Boudet J., Frisk M., Barthouil P., Borderies P., Meyer P. Effect of zolpidem during sleep on ventilation and cardiovascular variables in normal subjects - Fundam Clin Pharmacol, 1993 1993;7(6):305-10

12. Dujardin K., Guieu J.D., Leconte-Lambert C., Leconte P., Borderies P., de La Giclais B. Comparison of the effects of zolpidem and flunitrazepam on sleep structure and daytime cognitive functions. A study of untreated insomniacs - Pharmacopsychiatry 1998 Jan; 31(1):14-8

13. MacFarlane J., Morin C. M., Montplaisir J. Hypnotics in Insomnia: The Experience of Zolpidem - Clinical Therapeutics/Volume 36, Number 11, 2014

14. American Geriatrics Society 2015 Updated Beers Criteria for Potentially Inappropriate Medication Use in Older Adults - J Am Geriatr Soc, 2015 Nov;63(11):2227-46

15. Chen C.S., Huang M.F., Hwang T.J., Chen S.T., Ko C.H., Yen C.N., Chen T.T., Su P.W., Yeh Y.C., Lin J.J., Yen C.F. Clinical correlates of zolpidem-associated complex sleep-related behaviors: age effect J Clin Psychiatry, 2014 Nov;75(11)

16. Chen L.F., Lin C.E., Chou Y.C., Mao W.C., Chen Y.C., Tzeng N.S. A comparison of complex sleep behaviors with two short-acting and promoting sleep, but dosing the sleep side-effects of our medication: antidepressants, antihistaminergic drugs and neuroleptics may aggravate the RLS; SSRI antidepressants may induce insomnia complaints; benzodiazepines, barbiturates, muscle relaxants and opiates are able to lead to sleep breathing disorders; antidepressants, Z-drugs, neuroleptics, antihistamines may induce parasomnia behaviors, AED usually modify sleep macrostructure at the beginning of treatment.

Z-hypnosedative drugs in nonpsychotic patients - Neuropsychiatr Dis Treat, 2013; 9:1159-62

17. Burgess H.J., Revell V.L., Eastman C.I. A three pulse phase response curve to three milligrams of melatonin in humans $-\mathrm{J}$ Physiol, 2008 Jan 15;586(2):639-47

18. Mayer G., Wang-Weigand S., Roth-Schechter B., Lehmann R., Staner C., Partinen M. Efficacy and safety of 6-month nightly ramelteon administration in adults with chronic primary insomnia - Sleep, 2009 Mar;32(3):351-60

19. Mini L.J., Wang-Weigand S., Zhang J. Self-reported efficacy and tolerability of ramelteon $8 \mathrm{mg}$ in older adults experiencing severe sleep-onset difficulty - Am J Geriatr Pharmacother, 2007 Sep; $5(3): 177-84$

20. Vladimir Shvarts, Steve Chung, Epilepsy, antiseizure therapy and sleep cycle parameters - Epilepsy research and Treatment, 2013

21. G.L. Gigli, F. Placidi, M. Diomidi, Nocturnal sleep and daytime somnolence in untreated patients with temporal lobe epilepsy: changes after treatment with controlled-release Carbamazepine Epilepsia, vol.38, no.6, 1997

22. Diomedi M., Scalise A., Marciani MG., Romigi A., Gigli G.L., Placidi F. Effect of anticonvulsants on nocturnal sleep in epilepsy - Neurology, vol 54, 2000

23. Sadler M. Lamotrigine associated with insomnia - Epilepsia, vol.40, no.3, 1999

24. Arroyo S., Anhut H., Kugler R. Pregabaline add-on treatment: a randomized, double-blind, placebo-controlled, dose-response study in adults with partial seizures - Epilepsia, vol.45, no.1. 2004

25. Jain S.V., Glauser T.A. Effects of epilepsy treatments on sleep architecture and daytime sleepiness: an evidence-based review of objective sleep metrics - Epilepsia, 2014, 55(1): 26-37

26. Bell C., Vanderlinden H., Hiersemenzel R., Otoul C., Nutt D., Wilson S. The effects of levetiracetam on objective and subjective sleep parameters in healthy volunteers and patients with partial epilepsy - J Sleep Res, 2002, 11(3): 255-63

27. Charo G., Caletti MT., Provini F. Treatment of sleep-related eating disorder - Curr Treat Options Neurol, 2015, 17(8):361

28. Bonanni E., Galli R., Maestri M., Pizzanelli C., Fabbrini M., Manca M.L., ludice A., Murri L. Daytime sleepiness in epilepsy patients receiving topiramate monotherapy - Epilepsia 2004, 45(4): 333-7

29. Reife R., Pledger G., WU S.C. Topiramate as add-on therapy: pooled analysis of randomized controlled trials in adults - Epilepsia 2004, 41

30. Gillham R.A., Blacklaw J., McKee P.J., Brodie M.J. Effect of vigabatrin on sedation and cognitive function in patients with refractory epilepsy - J Neurol Neurosurg Psychatry, 1993, 56(12)

31. Raol I.H., Meti B.L. Effects of vigabatrin on sleep-wakefulness cycle in amygdala-kindled rats - Epilepsia, 2000, 41(12)

32. Hudson J.D., Guptill J.T., Byrnes W., Yates S.L., Williams P., D'Cruz O. Assessment of the effects of lacosamide on sleep parameters in healthy subjects - Seizure, 2015 Feb;25:155-9 
33. Giorgi F.S., Pizzanelli C., Pelliccia V., Di Coscio E., Maestri M, Guida M., lacopini E., ludice A., Bonanni E. A Clinical-EEG Study of Sleepiness and Psychological Symptoms in Pharmacoresistant Epilepsy Patients Treated with Lacosamide - Epilepsy Res Treat, 2013:593149

34. Lankford A., Rogowski R., Essink B., Ludington E., Heith Durrence H., Roth T. Efficacy and safety of doxepin $6 \mathrm{mg}$ in a four-week outpatient trial of elderly adults with chronic primary insomnia - Sleep Med, 2012 Feb;13(2):133-8

35. Chalon S., Pereira A., Lainey E., Vandenhende F., Watkin J.G., Staner L., Granier L.A. Comparative effects of duloxetine and desipramine on sleep EEG in healthy subjects - Psychopharmacology 2005 Feb;177(4):357-65

36. Zhang B., Hao Y., Jia F., Li X., Ren Y., Zhou P., Liu W., Wing Y.K. Sertraline and periodic limb movements during sleep: an 8-week open-label study in depressed patients with insomnia - Sleep Med, 2013 Dec;14(12):1405-12

37. Yang C., White D.P., Winkelman J.W. Antidepressants and periodic leg movements of sleep - Biol Psychiatry, 2005 Sep 15;58(6):510-4

38. Zhang B., Hao Y., Jia F., Tang Y., Li X., Liu W., Arnulf I. Sertraline and rapid eye movement sleep without atonia: an 8-week, open-label study of depressed patients - Prog Neuropsychopharmacol Biol Psychiatry 2013 Dec 2;47:85-92

39. Schenck C.H., Mahowald M.W., Kim S.W., O’Connor K.A., Hurwitz T.D. Prominent eye movements during NREM sleep and REM sleep behavior disorder associated with fluoxetine treatment of depression and obsessive-compulsive disorder - Sleep, 1992 Jun;15(3):226-35

40. Wang J., Greenberg H. Status cataplecticus precipitated by abrupt withdrawal of venlafaxine - J Clin Sleep Med, 2013 Jul 15;9(7):715-6

41. Simon D.K., Nishino S., Scammell T.E. Mistaken diagnosis of psychogenic gait disorder in a man with status cataplecticus ("limp man syndrome") - Mov Disord, 2004 Jul;19(7):838-40

42. Savarese M., Carnicelli M., Cardinali V., Mogavero M.P., Federico F. Subjective hypnotic efficacy of Trazodone and Mirtazapine in patients with chronic insomnia: a retrospective, comparative study - Arch Ital Biol. 2015 Sep 1;153(2-3):243-50

43. Camargos E.F., Louzada L.L., Quintas J.L., Naves J.O., Louzada F.M., Nóbrega O.T. Trazodone improves sleep parameters in Alzheimer disease patients: a randomized, double-blind, and placebo-controlled study - Am J Geriatr Psychiatry. 2014 Dec; 22(12):1565-74

44. Wiegand M.H., Galanakis P., Schreiner R. Nefazodone in primary insomnia: an open pilot study - Prog Neuropsychopharmacol Biol Psychiatry. 2004 Nov; 28(7):1071-8

45. Hublin C., Partinen M., Heinonen E.H., Puukka P., Salmi T. Selegiline in the treatment of narcolepsy - Neurology. 1994 Nov; 44(11):2095-101

46. Lazowski L.K., Townsend B., Hawken E.R., Jokic R., du Toit R., Milev R. Sleep architecture and cognitive changes in olanzapinetreated patients with depression: a double blind randomized placebo controlled trial - BMC Psychiatry. 2014 Jul 17;14:202

47. Khazaie H., Rezaie L., Darvishi F., Najafi F., Avis K. Treatment of paradoxical insomnia with atypical antipsychotic drugs. A comparison of olanzapine and risperidone - Neurosciences (Riyadh). 2013 Jan; 18(1):64-9

48. Cohrs S., Rodenbeck A., Guan Z., Pohlmann K., Jordan W., Meier A., Rüther E. Sleep-promoting properties of quetiapine in healthy subjects - Psychopharmacology (Berl). 2004 Jul;174(3):421-9

49. Wetter T.C., Brunner J., Bronisch T. Restless legs syndrome probably induced by risperidone treatment - Pharmacopsychiatry. 2002 May; 35(3):109-11

50. Mayer G., Benes H., Young P., Bitterlich M., Rodenbeck A. Modafinil in the treatment of idiopathic hypersomnia without long sleep time - a randomized, double-blind, placebo-controlled study J Sleep Res. 2015 Feb;24(1):74-81

51. Beck J., Hemmeter U., Brand S., Muheim F., Hatzinger M., Holsboer-Trachsler E. Modafinil reduces microsleep during partial sleep deprivation in depressed patients - J Psychiatr Res. 2010 Oct; 44(13):853-64
52. Schwartz J.R., Nelson M.T., Schwartz E.R., Hughes R.J. Effects of modafinil on wakefulness and executive function in patients with narcolepsy experiencing late-day sleepiness - Clin Neuropharmacol. 2004 Mar-Apr; 27(2):74-9

53. Davies M., Wilton L., Shakir S. Safety profile of modafinil across a range of prescribing indications, including off-label use, in a primary care setting in England: results of a modified prescription-event monitoring study - Drug Saf. 2013 Apr; 36(4):237-46

54. Mamelak M., Swick T., Emsellem H., Montplaisir J., Lai C., Black J. A 12-week open-label, multicenter study evaluating the safety and patient-reported efficacy of sodium oxybate in patients with narcolepsy and cataplexy - Sleep Med. 2015 Jan;16(1):52-8

55. Black J., Pardi D., Hornfeldt C.S., Inhaber N. The nightly use of sodium oxybate is associated with a reduction in nocturnal sleep disruption: a double-blind, placebo-controlled study in patients with narcolepsy - J Clin Sleep Med. 2010 Dec 15; 6(6):596-602

56. Leu-Semenescu S., Nittur N., Golmard J.L., Arnulf I. Effects of Pitolisant, a histamine $\mathrm{H} 3$ inverse agonist, in drug resistant idiopathic and symptomatic hypersomnia: a chart review - Sleep Medicine, 2014 Jun; 15(6):681-7

57. American Academy of Sleep Medicine Clinical Practice Guideline, The Treatment of Restless Legs Syndrome and Periodic Limb Movement Disorder in Adults - An Update for 2012: Practice Parameters with an EvidenceBased Systematic Review and Meta-Analyses - SLEEP 2012; 35(8):1039-1062.

58. Manconi M., Ferri R., Zucconi M., Clemens S., Giarolli L., Bottasini V., Ferini-Strambi L. Preferential D2 or preferential D3 dopamine agonists in restless legs syndrome - Neurology. $2011 \mathrm{Jul}$ 12; 77(2):110-7

59. Jama L., Hirvonen K., Partinen M., Alakuijala A., Hublin C., Tamminen I., Koester J., Reess J. A dose-ranging study of pramipexole for the symptomatic treatment of restless legs syndrome: polysomnographic evaluation of periodic leg movements and sleep disturbance - Sleep Med. 2009 Jun; 10(6):630-6

60. Voon V., Hassan K., Zurowski M., Duff-Canning S., de Souza M., Fox S., Lang A.E., Miyasaki J. Prospective prevalence of pathologic gambling and medication association in Parkinson disease Neurology. 2006 Jun 13;66(11):1750-2

61. Murphy P.J., Badia P., Myers B.L., Boecker M.R., Wright K.P. Jr. Nonsteroidal anti-inflammatory drugs affect normal sleep patterns in humans - Physiol Behav. 1994 Jun; 55(6):1063-6

62. Panagiotou I., Mystakidou K. Non-analgesic effects of opioids: opioids' effects on sleep - Curr Pharm Des. 2012;18(37)

63. Dimsdale J., Norman D., DeJardin D. The Effect of Opioids on Sleep Architecture - Journal of Clinical Sleep Medicine, Vol. 3, No. 1, 2007

64. Webster L.R., Choi Y., Desai H., Webster L., Grant B.J. Sleep-disordered breathing and chronic opioid therapy - Pain Med. 2008 May-Jun; 9(4):425-32

65. Herring W.J., Connor K.M., Ivgy-May N., Snyder E., Liu K., Snavely D.B., Krystal A.D., Walsh J.K., Benca R.M., Rosenberg R., Sangal R.B., Budd K., Hutzelmann J., Leibensperger H., Froman S., Lines C., Roth T., Michelson D. Suvorexant in Patients With Insomnia: Results From Two 3-Month Randomized Controlled Clinical Trials - Biol Psychiatry, 2016, 79(2) 136-48

66. Hagan J.J., Leslie R.A., Patel S., Evans M.L., Wattam T.A., Holmes $\mathrm{S}$. et al. Orexin A activates locus coeruleus cell firing and increases arousal in the rat - Proc Natl Acad Sci USA. 1999 Sep 14; 96(19):10911-6

67. Nishino S., Mignot E. Pharmacological aspects of human and canine narcolepsy - Prog Neurobiol. 1997 May; 52(1):27-78

68. Raskind M.A., Peterson K., Williams T., Hoff D.J., Hart K., Holmes H., et al. A trial of prazosin for combat trauma PTSD with nightmares in active-duty soldiers returned from Iraq and Afghanistan - Am J Psychiatry. 2013 Sep;170(9):1003-10

69. Empson J.A., Purdie D.W. Effects of sex steroids on sleep - Ann Med. 1999 Apr; 31(2):141-5

70. Antonijevic I.A., Stalla G.K., Steiger A. Modulation of the sleep electroencephalogram by estrogen replacement in postmenopausal women - Am J Obstet Gynecol. 2000 Feb;182(2):277-82 
71. Montplaisir J., Lorrain J., Denesle R., Petit D. Sleep in menopause: differential effects of two forms of hormone replacement therapy Menopause. 2001 Jan-Feb;8(1):10-6

72. Saletu-Zyhlarz G., Anderer P., Gruber G., Mandl M., Gruber D., Metka M. et al. Insomnia related to postmenopausal syndrome and hormone replacement therapy: sleep laboratory studies on baseline differences between patients and controls and double-blind, placebo-controlled investigations on the effects of a novel estrogenprogestogen combination (Climodien, Lafamme) versus estrogen alone - J Sleep Res. 2003 Sep;12(3):239-54

Conflict of interest: none declared Financial support: none declared 